\title{
Ideal, First-kind Measurements in a Proposition-State Structure
}

\author{
G. Cassinelli and E. G. Beltrametti \\ Istituto di Scienze Fisiche, Università di Genova, I.N.F.N., Sezione di Genova, \\ Genova, Italy
}

Received May 9, 1974

\begin{abstract}
Let $\mathscr{L}$ be an orthomodular lattice and $\mathscr{S}$ a strongly ordering set of probability measures on $\mathscr{L}$ such that supports of measures exist in $\mathscr{L}$. Then we show the existence of a set of mappings of $\mathscr{S}$ into $\mathscr{S}$ that are physically interpretable as ideal, first-kind measurements.
\end{abstract}

\section{1.}

In the conventional formulation [1-3] of the so called logic of quantum mechanics the basic mathematical structure associated to a physical system is the pair $(\mathscr{L}, \mathscr{S})$ where $\mathscr{L}$ is the set of propositions (yes-no experiments), $\mathscr{S}$ the set of states; each $\alpha \in \mathscr{S}$ defines on $\mathscr{L}$ a probability measure $\alpha: \mathscr{L} \rightarrow[0,1]$, and $\alpha(a), a \in \mathscr{L}$, is interpreted as the probability of the yes answer of $a$, when the initial state of the system is $\alpha . \mathscr{L}$ is given the appropriate structure of orthomodular lattice by means of suitable axioms which elude any requirement on the transformations of the state of the system caused by the measurement of $a$ : the usual postulates of quantum theory of measurement are considered as independent from the structure of $\mathscr{L}$ (for details and further bibliography we refer to [4]).

Pool $[5,6]$ has suggested an alternative approach which uses as basic mathematical structure the proposition-state-operation triple $(\mathscr{L}, \mathscr{S}, \Omega)^{1}$, where the operation $\Omega_{a} \in \Omega$ associated to $a \in \mathscr{L}$ is understood as the transformation of the state of the system induced by an ideal, first-kind measurement (with yes answer) of the proposition $a$. By use of the postulates of quantum theory of measurement and of the remarkable connections between orthomodular lattices and Baer*-semigroups, he deduces for $\mathscr{L}$ the structure of orthomodular lattice (see also [7]).

In this paper, we shall examine the possibility of reversing the Pool approach: we are going to study whether the assumption of a $(\mathscr{L}, \mathscr{S})$ structure, with $\mathscr{L}$ orthomodular lattice, is sufficient to deduce the

${ }^{1}(\mathscr{L}, \mathscr{S})$ is denoted by Pool as $(\mathscr{E}, \mathscr{S}, P)$ and called an event-state structure; $(\mathscr{L}, \mathscr{P}, \Omega)$ is denoted as $(\mathscr{E}, \mathscr{S}, P, \Omega)$ and called an event-state-operation structure. 
existence of a set of transformations of $\mathscr{S}$ into $\mathscr{S}$ which admit the physical interpretation of ideal, first-kind measurements, hence the existence of operations in the sense of Pool.

2.

We sum up several usual assumptions about $\mathscr{L}$ and $\mathscr{S}$ by the following

Axiom 1. $\mathscr{L}$ is a complete orthomodular lattice, $\mathscr{S}$ is a strongly ordering, $\sigma$-convex set of probability measures on $\mathscr{L}$.

Here, and in the sequel, the definitions and the notations about lattices are from [8], the definitions about probability measures on orthomodular posets are from $[5,6]$.

We say that $a \in \mathscr{L}$ is support of $\alpha \in \mathscr{S}$ when

$$
\alpha(b)=0, \quad b \in \mathscr{L} \Leftrightarrow a \perp b .
$$

For every $\alpha \in \mathscr{S}$ there exists at most one $a \in \mathscr{L}$ such that $a$ is support of $\alpha$ : we shall denote such element, when it exists, by $\sigma(\alpha)$. We refer to [6] for properties of supports: the following will be used

$$
\begin{aligned}
& \{a \in \mathscr{L}: a \perp \sigma(\alpha)\}=\{a \in \mathscr{L}: \alpha(a)=0\}, \\
& \{a \in \mathscr{L}: \sigma(\alpha) \leqq a\}=\{a \in \mathscr{L}: \alpha(a)=1\} .
\end{aligned}
$$

Then we adopt, as in [6], the following

Axiom 2. If $\alpha \in \mathscr{S}$ then the support of $\alpha$ exists in $\mathscr{L}$. If $a \in \mathscr{L} \backslash\{\mathbf{0}, \mathbf{1}\}$ then there exists $\alpha \in \mathscr{S}$ such that $a=\sigma(\alpha)$.

Hence it is defined a surjective mapping

$$
\alpha \mapsto \sigma(\alpha): \mathscr{S} \rightarrow \mathscr{L} \backslash\{\mathbf{0}, \mathbf{1}\}
$$

which is always canonically decomposable in the composition of a surjective mapping $\omega$ of $\mathscr{S}$ onto $\mathscr{S} / \sigma$ (the set of the equivalence classes of the states with the same support) with a bijection $f$ of $\mathscr{S} / \sigma$ onto $\mathscr{L} \backslash\{\mathbf{0 , 1}\}$. Thus $f$ defines an one-to-one correspondence between $\mathscr{S} / \sigma$ and $\mathscr{L} \backslash\{\mathbf{0}, \mathbf{1}\}$ such that every element of $\mathscr{S} / \sigma$ corresponds to the common support of the states belonging to that element.

Let $S(\mathscr{L})$ be the Baer*-semigroup of residuated mappings (or emimorphisms) of $\mathscr{L}^{2}$, let $P^{\prime}(S(\mathscr{L}))$ be the lattice of the closed projections of $S(\mathscr{L})$. For every $a \in \mathscr{L}$, let

$$
\varphi_{a}(b)=\left(b \vee a^{\perp}\right) \wedge a, \quad b \in \mathscr{L} .
$$

${ }^{2}$ We refer to $[5,6,8]$ for definitions, properties and bibliography on Baer *-semigroups, residuated mappings of orthomodular lattices, and their connections. 
$\varphi_{a}$ is an element of $P^{\prime}(S(\mathscr{L}))$; conversely every element of $P^{\prime}(S(\mathscr{L}))$ is of the form $\varphi_{a}$ for some $a \in \mathscr{L}$.

The pair $(\mathscr{L}, \mathscr{S})$ will be definitely assumed to satisfy Axioms 1 and 2: we shall call it the $(\mathscr{L}, \mathscr{S})$ structure.

Lemma 1. For any given $a \in \mathscr{L}$ there exists a unique mapping $\omega_{a}: \mathscr{S} \rightarrow \mathscr{S} / \sigma$ such that

(i) the following diagram is commutative

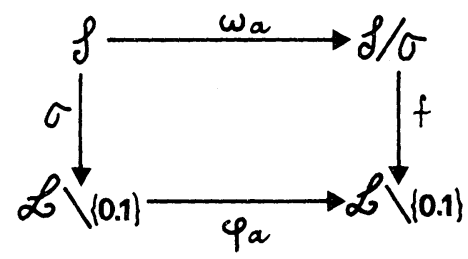

(ii) the domain $\mathscr{D}\left[\omega_{a}\right]$ of $\omega_{a}$ is given by

$$
\mathscr{D}\left[\omega_{a}\right]=\{\alpha \in \mathscr{S}: \sigma(\alpha) \not \alpha a\} .
$$

Proof. Given $a \in \mathscr{L}, \alpha \in \mathscr{S}$, a known property of closed projections of $S(\mathscr{L})$ ensures that $\varphi_{a}(\sigma(\alpha)) \neq \mathbf{0}$ if and only if $\sigma(\alpha) \not a$. Thus, if $\alpha \in\{\beta \in \mathscr{S}: \sigma(\beta) \not a\}, \varphi_{a}(\sigma(\alpha)) \in \mathscr{L} \backslash\{\mathbf{0}, \mathbf{1}\}$. Since $f$ is a bijection it is well defined $f^{-1}\left(\varphi_{a}(\sigma(\alpha))\right) \in \mathscr{S} / \sigma$. Then we put

$$
\omega_{a}(\alpha)=f^{-1}\left(\varphi_{a}(\sigma(\alpha))\right) \text { if } \sigma(\alpha) \not \alpha a .
$$

In this way we have defined a mapping of $\mathscr{S}$ into $\mathscr{S} / \sigma$ whose domain is $\mathscr{D}\left[\omega_{a}\right]=\{\alpha \in \mathscr{S}: \sigma(\alpha) \not a\}$. Clearly $\omega_{a}$ makes the diagram commutative and is uniquely determined by the Properties (i) and (ii). Q.E.D.

Lemma 2. Given $a \in \mathscr{L}, \alpha \in \mathscr{D}\left[\omega_{a}\right]$,

(i) if $\alpha(a)=1$ then $\omega_{a}(\alpha)=\{\alpha\}$, where $\{\alpha\}$ is the equivalence class of $\alpha$ in $\mathscr{S} / \sigma$;

(ii) if $\beta \in \omega_{a}(\alpha)$ then $\beta(a)=1$.

Proof. (i) $\alpha(a)=1$ implies $\sigma(\alpha) \leqq a$, hence $\sigma(\alpha) C a$, hence $\sigma(\alpha) C a^{\perp}$ [in an orthomodular lattice we say that $a$ commutes with $b$, and we write $a C b$ when $\left.a=(a \wedge b) \vee\left(a \wedge b^{\perp}\right)\right]$. Therefore $a, a^{\perp}, \sigma(\alpha)$ form a distributive triple so that

$$
\begin{aligned}
\varphi_{a}(\sigma(\alpha))= & \left(\sigma(\alpha) \vee a^{\perp}\right) \wedge a=(\sigma(\alpha) \wedge a) \vee\left(a^{\perp} \wedge a\right)=\sigma(\alpha), \\
& \text { whence } f^{-1}\left(\varphi_{a}(\sigma(\alpha))\right)=\omega_{a}(\alpha)=\{\alpha\} .
\end{aligned}
$$

(ii) Owing to the commutativity of the diagram of Lemma 1 we have $f\left(\omega_{a}(\alpha)\right)=\varphi_{a}(\sigma(\alpha)), \forall \alpha \in \mathscr{D}\left[\omega_{a}\right]$, hence $f\left(\omega_{a}(\alpha)\right) \leqq a$ for $\varphi_{a}$ projects $\mathscr{L}$ onto the sublattice $[\mathbf{0}, a]$. If $\beta \in \omega_{a}(\alpha)$, by the definition of $f$ we get

$$
\sigma(\beta)=f\left(\omega_{a}(\alpha)\right) \leqq a, \text { whence } \beta(a)=1 . \quad \text { Q.E.D. }
$$


Lemma 3. If $a, b \in \mathscr{L}, a C b, \alpha \in \mathscr{D}\left[\omega_{a}\right], \beta \in \omega_{a}(\alpha)$ then

(i) $\alpha(b)=1$ implies $\beta(b)=1$;

(ii) $\beta(b)=\beta(a \wedge b)$.

Proof. (i) $\alpha(b)=1$ implies $\sigma(\alpha) \leqq b$, hence $\varphi_{a}(\sigma(\alpha)) \leqq \varphi_{a}(b)$ for $\varphi_{a}$ is monotone (as every residuated mapping). From $a C b$ it follows that $a, a^{\perp}, b$ form a distributive triple, hence

$$
\varphi_{a}(b)=\left(b \vee a^{\perp}\right) \wedge a=(b \wedge a) \vee\left(a^{\perp} \wedge a\right)=b \wedge a .
$$

The commutativity of the diagram of Lemma 1 ensures that if $\beta \in \omega_{a}(\alpha)$ then $\sigma(\beta)=\varphi_{a}(\sigma(\alpha))$. Therefore $\sigma(\beta) \leqq \varphi_{a}(b)=b \wedge a \leqq b$, whence $\beta(b)=1$.

(ii) We write $a C b$ in the form $b=(b \wedge a) \vee\left(b \wedge a^{\perp}\right)$ and remark that $\left(b \wedge a^{\perp}\right)^{\perp}=b^{\perp} \vee a \geqq b \wedge a$; thus $b \wedge a$ and $b \wedge a^{\perp}$ are orthogonal so that

$$
\beta(b)=\bar{\beta}(b \wedge a)+\beta\left(b \wedge a^{\perp}\right)=\beta(b \wedge a)+1-\beta\left(b^{\perp} \vee a\right) .
$$

On account of Lemma 2 (ii) we have $\beta\left(b^{\perp} \vee a\right)=1$ for $b^{\perp} \vee a \geqq a$; therefore $\beta(b)=\beta(b \wedge a)$.

Q.E.D.

Theorem 1. For every $a \in \mathscr{L}$, there exists at least one mapping $\Omega_{a}: \mathscr{S} \rightarrow \mathscr{S}$ such that

(A) $\Omega_{a}$ has domain $\mathscr{D}\left[\Omega_{a}\right]=\mathscr{D}\left[\omega_{a}\right]$,

(B) if $\alpha \in \mathscr{D}\left[\Omega_{a}\right]$, and $\alpha(a)=1$ then $\Omega_{a}(\alpha)=\alpha$,

(C) if $\alpha \in \mathscr{D}\left[\Omega_{a}\right]$, and $\beta=\Omega_{a}(\alpha)$ then $\beta(a)=1$,

(D) if $\alpha \in \mathscr{D}\left[\Omega_{a}\right], \beta=\Omega_{a}(\alpha), b \in \mathscr{L}, a C b$, and $\alpha(b)=1$ then $\beta(b)=1$,

(E) if $\alpha \in \mathscr{D}\left[\Omega_{a}\right], \beta=\Omega_{a}(\alpha), b \in \mathscr{L}$, and $a C b$ then $\beta(b)=\beta(a \wedge b)$,

(F) if $\alpha \in \mathscr{D}\left[\Omega_{a}\right]$, and $\beta=\Omega_{a}(\alpha)$ then $\sigma(\beta)=\varphi_{a}(\sigma(\alpha))$.

Proof. We notice that $\mathscr{S} / \sigma$ is a partition of $\mathscr{S}$ (in disjoint classes) and we make use of the so called choice axiom (see, e.g., [9]): composing $\omega_{a}$ with any choice function which maps $\omega_{a}(\alpha) \in \mathscr{S} / \sigma$ into some single state belonging to $\omega_{a}(\alpha)$ we get, for every $a \in \mathscr{L}$, a mapping $\Omega_{a}$ of $\mathscr{S}$ into $\mathscr{S}$. By construction $\Omega_{a}$ fits $(A)$. On account of Lemma 2(i), and remarking that $\alpha \in\{\alpha\}$, we are allowed to adopt a choice function which, whenever $\alpha(a)=1$, maps $\omega_{a}(\alpha)$ into $\alpha$ itself: thus $\Omega_{a}$ satisfies $(B)$. The Properties $(C),(D),(E),(F)$ are then direct consequences of Lemmas 2 (ii), 3 (i), 3 (ii), 1 (i), respectively.

Q.E.D.

3.

The previous theorem answers affirmatively the problem rised at the end of Section 1 ; the $(\mathscr{L}, \mathscr{S})$ structure (equipped with the Axioms 1 and 2 ) is sufficient to deduce, for every $a \in \mathscr{L}$, the existence of a mapping $\Omega_{a}$ of $\mathscr{S}$ into $\mathscr{S}$ which admits the following interpretation: if the initial state of the system is $\alpha$ then $\Omega_{a}(\alpha)$ is the final state of the system after an ideal, first-kind measurement of $a$ which has given the yes answer. Let us examine briefly the Properties $(A)-(F)$ of that theorem. 
Since the condition $\sigma(\alpha) \not a$ (occuring in $\mathscr{D}\left[\Omega_{a}\right]$ ) is equivalent to $\alpha(a) \neq 0$, we rephrase $(A)$ by saying that $\Omega_{a}$ is defined for every state $\alpha$ which has a non vanishing probability $\alpha(a)$ of giving the yes answer of the yes-no experiment $a$. Then $(B)$ and $(C)$ correspond to the definition of first kind measurement [10]. The commutativity relation $C$ previously used is fully equivalent to the physical notion of compatible experiments $[3,7,8]$. Thus the Property $(D)$ corresponds to the commonly accepted definition of ideal measurement. When $\beta=\Omega_{a}(\alpha)$ and $a C b$ the Property $(E)$ suggests for $\beta(b)$ the interpretation of conditional probability of compatible events; however, to guarantee that interpretation one should require, according to conventional probability theory, one further property, viz:

(G) if $a, b \in \mathscr{L}, a \geqq b, \alpha \in \mathscr{D}\left[\Omega_{a}\right]$, and $\beta=\Omega_{a}(\alpha)$ then $\beta(b)=\frac{\alpha(b)}{\alpha(a)}$.

The Property $(F)$, which determines uniquely the support of $\beta=\Omega_{a}(\alpha)$ from the support of $\alpha$, is the essential step to equip $\mathscr{L}$ with the covering property [6].

We come now to a comparison with the assumptions occuring in the Pool approach. The Properties $(A),(B)$, and $(C)$ coincide, respectively, with his Axioms II.1, II.2, and II.3. The Property $(D)$ goes beyond the Pool axioms: actually he uses an alternative definition of ideal measurement resting on the Properties $(E)$ and $(G)$, which coincide, respectively, with his Axioms II.7 and II.6. However, these two axioms enter into the Pool approach only as instruments to prove a property weaker than $(F)$, viz:

$$
\text { if } \alpha \in \mathscr{D}\left[\Omega_{a}\right] \text {, and } \beta=\Omega_{a}(\alpha) \text { then } \sigma(\beta) \leqq \varphi_{a}(\sigma(\alpha)) \text {. }
$$

This inequality can also be proved by use of the characterization $(D)$ of ideal measurement $[4,7]$ without any reference to $(E)$ and $(G)$; anyhow it is used by Pool only as a hint for adopting the stronger Property $(F)$ which coincides with his Axiom II.8.

Thus we have to conclude that the $(\mathscr{L}, \mathscr{S})$ structure contains into itself the relevant properties of Pool's operations ${ }^{3}$ with just one significant exception: the unicity of the ideal, first-kind measurement associated to $a \in \mathscr{L}$, (which is necessary to deduce the orthomodular lattice properties of $\mathscr{L}$ from the proposition-state-operation structure).

However, in our approach, the problem of the unicity of $\Omega_{a}$ has connections with the further hypotheses, about the atomicity of $\mathscr{L}$ and the pure states of $\mathscr{S}$, which are needed to introduce the covering property (called semimodularity in [6]). For the last we shall refer to the definition: $\mathscr{L}$ has the covering property if $\varphi_{a}(p)$ is an atom for every $a \in \mathscr{L}$ and

\footnotetext{
${ }^{3}$ The Axioms II.4 and II.5 of Pool evade the framework of the present analysis.
} 
every atom $p \in \mathscr{L}$ such that $p \Varangle a^{\perp}$. This definition is equivalent [11] to the one found in [8]. Then we have:

Theorem 2. If, in the structure $(\mathscr{L}, \mathscr{S}), \mathscr{L}$ is atomic and $\sigma$ determines a bijective mapping between the atoms of $\mathscr{L}$ and the pure states of $\mathscr{S}$, then

(i) the covering property of $\mathscr{L}$ implies that the restriction of $\Omega_{a}$ to the pure states of $\mathscr{D}\left[\Omega_{a}\right]$ is uniquely determined by a and transforms pure states into pure states;

(ii) $\mathscr{L}$ has the covering property and the restriction of $\Omega_{a}$ to the pure states of $\mathscr{D}\left[\Omega_{a}\right]$ is uniquely determined by a if $\Omega_{a}$ transforms pure states into pure states.

Proof. (i) Due to the covering property $\varphi_{a}(\sigma(\alpha))$ is an atom; by $(F)$ of Theorem 1 this atom is the support of $\beta=\Omega_{a}(\alpha)$. Hence this state is pure and uniquely determined by $a$ and $\alpha$.

(ii) By hypothesis, if $\alpha \in \mathscr{D}\left[\Omega_{a}\right]$ is pure, also $\Omega_{a}(\alpha)$ is pure, hence $\varphi_{a}(\sigma(\alpha))$ is an atom, by $(F)$ of Theorem 1 . This is equivalent to say that $\varphi_{a}(p)$ is an atom whenever $p \in \mathscr{L}$ is an atom such that $p \npreceq a^{\perp}$. The uniqueness of $\beta=\Omega_{a}(\alpha)$ follows from $\sigma(\beta)=\varphi_{a}(\sigma(\alpha))$.

Q.E.D.

The hypotheses of Theorem 2 about atomicity and pure states are equivalent to the ones adopted in [6] (see Axiom I.9 and Theorem II.1). We conclude that also the connections between covering property and pure operations are contained, in a natural way, into the $(\mathscr{L}, \mathscr{S})$ structure.

The role of the unicity of the ideal, first-kind measurement associated to $a \in \mathscr{L}$ has been particularly studied by Ochs [12]. He restricts $\mathscr{S}$ to the set $\mathscr{S}^{\prime}$ of pure states, assumes $\mathscr{L}$ to be an atomic orthomodular lattice with a bijective mapping of $\mathscr{S}^{\prime}$ onto the set of the atoms of $\mathscr{L}$, postulates the existence and the unicity of an ideal, first-kind measurement [i.e. the Properties $(A),(B),(C),(D)$ of Theorem 1 with $\Omega_{a}$ replaced by $\tau_{a}$ ], and proves the covering property [i.e. the Property $(F)$ of Theorem 1 with $\Omega_{a}$ replaced by $\tau_{a}$ ]. On account of Theorem 2 , the restriction of $\Omega_{a}$ to the pure states coincides with $\tau_{a}$. Thus, when dealing with pure states, the requirement of unicity of the ideal, first-kind measurement is equivalent to adopt our construction of $\Omega_{a}$, made explicit by $(F)$ of Theorem 1.

\section{References}

1. Piron, C.: Helv. Phys. Acta 37, 439-468 (1964)

2. Jauch, J.M.: Foundations of quantum mechanics. Reading, Mass.: Addison-Wesley 1968

3. Varadarajan, V.S.: Geometry of quantum theory, Vol. 1. Princeton: Van Nostrand 1968

4. Beltrametti, E. G., Cassinelli, G.: Z. Naturforsch. 28a, 1516-1530 (1973)

5. Pool, J.C. T.: Commun. math. Phys. 9, 118-141 (1968) 
6. Pool, J.C.T.: Commun. math. Phys. 9, 212-228 (1968)

7. Beltrametti,E.G., Cassinelli, G.: On the structure of the proposition lattice associated with quantum systems. In: Atti del colloquio internazionale sulle teorie combinatorie. Roma: Acc. Naz. Lincei, in press

8. Maeda,F., Maeda,S.: Theory of symmetric lattices. Berlin-Heidelberg-New York: Springer 1970

9. Dieudonné, J.: Foundations of modern analysis. New York: Academic Press 1969 (in particular p. 6)

10. Pauli, W.: Die allgemeinen Prinzipien der Wellenmechanik. In: Handbuch der Physik, Vol. 1, Part 1, pp. 1-168. Berlin-Göttingen-Heidelberg: Springer 1958

11. Schreiner,E. A.: Pacific J. Math. 19, 519-528 (1966)

12. Ochs. W.: Commun. math. Phys. 25, 245-252 (1972)

Communicated by R. Haag

G. Cassinelli

E. G. Beltrametti

Università di Genova

Istituto di Scienze Fisiche

Viale Benedetto XV, 5

I-16132 Genova, Italy 
